\title{
Colloidal Stability of Aqueous Suspensions of Polymer-Coated Iron Oxide Nanorods: Implications for Biomedical Applications
}

J. A. Marins ${ }^{1 *}$, T. Montagnon ${ }^{1}$, H. Ezzaier ${ }^{1}$, Ch. Hurel ${ }^{1}$, O. Sandre ${ }^{2}$, D. Baltrunas ${ }^{3}$, K.Mazeika $^{3}$, A.Petrov ${ }^{4}$, and P. Kuzhir ${ }^{1 *}$

${ }^{1}$ University Côte d'Azur, CNRS UMR 7010 Institute of Physics of Nice, Parc Valrose 06108 Nice, France

${ }^{2}$ University of Bordeaux, CNRS UMR 5629, Laboratoire de Chimie des Polymères Organiques, ENSCBP 16 avenue Pey Berland 33607 Pessac, France

${ }^{3}$ Nuclear Gamma Resonance Laboratory, State research institute Center for Physical Sciences and Technology, Savanorių ave. 231, LT-02300 Vilnius, Lithuania

${ }^{4}$ Cryogenic Research Division, Scientific-Practical Materials Research Centre of the National Academy of Sciences of Belarus, P. Brovka Str., 19, 220072 Minsk, Belarus

\section{ABSTRACT}

Iron oxide nanorods are considered to be very promising platforms for biomedical applications, such as magnetic hyperthermia, magnetic resonance imaging or immunoassays based on magneto-optical effects. However, their efficient colloidal stabilization is challen-
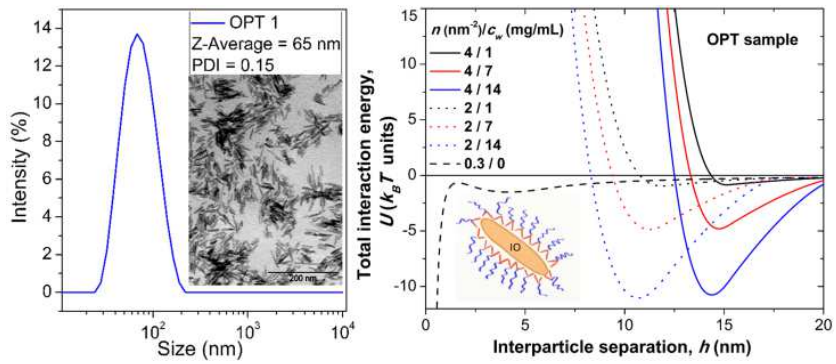

ging, and colloidal aggregation could lead to the total loss of their performance. This work is focused on synthesis and colloidal stabilization of iron oxide nanorods of an average length and diameter $L \times d=31 \times 6 \mathrm{~nm}$, synthetized by hydrolysis of iron (III) salt followed by reduction of the obtained akaganeite to iron oxide in a microwave reactor. Synthesized nanorods exhibited a weak ferrimagnetic behavior with remnant magnetization $M_{R} \sim 3 \mathrm{emu} / \mathrm{g}$ and saturation magnetization $M_{S} \sim 13 \mathrm{emu} / \mathrm{g}$. The nanorods were dispersed in water after adsorption on their surface of three different polymers - linear bisphosphonate-polyethylene glycol (PEG) molecules (denoted OPT), polymethacrylate backbone / PEG side chains comb polymer (denoted PCP) (both with PEG brushes extended towards the solvent and having molecular weight $M_{w} \sim 3000 \mathrm{~g} / \mathrm{mol}$ ) and polyacrylic sodium salt (PAA, $M_{w} \sim 15000 \mathrm{~g} / \mathrm{mol}$ ). Experiments and theoretical evaluation of the interaction potential shows that increasing polymer grafting density on the nanorod surface as well as decreasing concentration of nonadsorbed polymer improve the nanorod colloidal stability. The best stability is obtained on an optimal range of weight ratio of the added polymer to the nanorods between 0.5 and 1.6 $\mathrm{mg} / \mathrm{mg}$. Higher grafting density reached with OPT polymer with a bisphosphonate terminal group (2-4 $\mathrm{nm}^{-2}$ ) allows much better stability than using multiple adsorption with PCP (0.2$0.4 \mathrm{~nm}^{-2}$ ) or PAA. Even though the nanorods are still subject to some aggregation (effective hydrodynamic diameter $\sim 60 \mathrm{~nm}$, as compared to their TEM size $L \times d=31 \times 6 \mathrm{~nm}$ ), significant progress towards understanding their colloidal stability was achieved.

KEYWORDS: magnetic nanoparticles; colloidal stability; polymer coating

\section{INTRODUCTION}

During last decades, scientific research related to synthesis, functionalization and colloidal stability of magnetic nanobeads has gained a growing popularity thanks to their biomedical applications ${ }^{1,2}$. Tuning the nanoparticle shape from spherical (nanobeads) to 
cylindrical (nanorods) has allowed a considerable improvement of performances of many biomedical applications. Due to enhanced magnetic properties and increased length-scale of the locally induced magnetic field, nanorods have shown stronger MRI contrast-enhancing effect $^{3-5}$, better separation efficiency in immuno-magnetic cell separation ${ }^{6}$ and stronger specific adsorption rate in magnetic hyperthermia ${ }^{7}$. On the other hand, the rod-like particle shape gives rise to several specific biomedical applications hardly possible with spherical particles. In particular, they have been successfully used for mechanical destruction of tumor cells under low-frequency applied magnetic fields ${ }^{8-10}$. As another example, hemozoin and $\beta$ haematin nanorods (naturally produced by the human body) are markers of malaria decease and have been successfully detected by magnetic field induced optical dichroism of blood samples contaminated by malaria parasites ${ }^{11}$. Recently, a robust method of biological molecules detection has been proposed on the basis of optical ${ }^{12,13}$ or magneto-optical ${ }^{14}$ detection of nanorod rotation in response to the applied rotating magnetic field and as a function of the amount of the biomolecules captured by the nanorod surface.

Among the different routes to iron oxide nanorods for biomedical applications listed in a recent review paper ${ }^{15}$, one common technique is a two-step synthesis consisting in preparation first of akaganeite $(\beta-\mathrm{FeOOH})$ rods (iron oxyhydroxide with weak paramagnetic properties at room temperature) followed by a reduction to ferrimagnetic iron oxide (magnetite or maghemite) ${ }^{16}$. Nanosized akaganeite rods are obtained through the hydrolysis of iron chloride $\left(\mathrm{FeCl}_{3}\right)$ in mild acidic or alkaline conditions in the presence of size capping agents such as dopamine ${ }^{17}$, polyethylimine $(\mathrm{PEI})^{4,18}$ or oleic acid ${ }^{7}$. Akaganeite nanorods are then reduced to the ferrimagnetic iron oxide phase (presumably magnetite) while keeping the nanorod shape and size using various reducing agents, such as oleylamine ${ }^{4}$, ethylene glycol $(\mathrm{EG})^{19}$ or hydrazine ${ }^{20}$.

Successful use of iron oxide nanorods in aforementioned applications is only possible if they do not aggregate under colloidal or magnetic forces in the suspending liquid. Colloidal stabilization of nanorods in an aqueous solvent appears to be much more difficult than that of nanobeads because van der Waals interaction is much stronger for nanorods since it makes them stack side-by-side with an effective contact area much larger than for nanobeads. Thus, electron microscopy pictures of the synthesized iron oxide nanorods often reveal presence of nanorod bundles $4,5,7,17,18$, even if appropriate polymers or surfactants are grafted on their surface to ensure steric repulsion ${ }^{4,5,7}$, except for recently used poly(MPS) homopolymer ${ }^{21}$ to cover micron-sized rods, for which colloidal forces are of less importance.

Such lateral aggregation of nanonorods reduces their effective length-to-diameter ratio, which is expected to decrease significantly their specific area, MRI-contrast enhancing effect and magneto-optic response. Despite such technological challenge, detailed study of the colloidal stability of iron oxide nanorods is still missing in literature. The main objective of this paper is realizing stable dilute water dispersions of iron oxide nanorods whose length remains below $50 \mathrm{~nm}$ and understanding the mechanisms leading to either aggregation or colloidal stabilization of these dispersions.

To this purpose, we synthetized iron oxide nanorods slightly modifying the aforementioned two-step method. The synthesized nanorods were then dispersed in distilled water using three different polymers or polyelectrolytes, such as linear poly(sodium acrylate) (denoted as PAA), comb-like poly (sodium methacrylate)-co-poly(oligoethyleneglycol methyl ether methacrylate) and di-functional N, N'-di(methylphosphonic acid) amino poly(ethylene glycol). The choice of these polymers was dictated by their commercial availability in addition to the fact that similar polymers were commonly employed as stabilizers of iron oxide nanobeads with superior performance both in vitro or in vivo, respectively for linear $\mathrm{PAA}^{22}$, poly(methacrylate) grafted with polyethylene glycol (PEG) chains and complexing side-groups ${ }^{23,24}$, and PEGs with a diphosphonic acid anchoring end-group ${ }^{25-31}$. According to 
the structures of these polymers (linear vs. comb-like and weak carboxylate vs. strong phosphonate ligands), the resulting adsorbed layers of these molecules may have different morphologies (loop and train or brush) allowing us to compare their performance for colloidal stabilization purposes. The quality of the nanorods dispersion was studied by transmission electron microscopy (TEM), cryoTEM and dynamic light scattering (DLS) as a function of the solvent $\mathrm{pH}$ and the amount and nature of the added polymer. This experimental study is completed by theoretical estimates of the interaction energies between nanorods, allowing a discussion on their colloidal stability and feedback for envisioned applications.

\section{EXPERIMENTAL SECTION}

2.1. Materials. Ferric chloride hexahydrate $\left(\mathrm{FeCl}_{3} \cdot 6 \mathrm{H}_{2} \mathrm{O}\right)$, poly(ethyeneimine) (PEI, molecular weight $M_{w}=25000 \mathrm{~g} / \mathrm{mol}$, branched), hydrazine $\left(\mathrm{N}_{2} \mathrm{H}_{4}\right)$ aqueous solution $(78-82 \%)$, sodium hydroxide $(\mathrm{NaOH})$ solution $(1 \mathrm{M})$, poly(sodium acrylate) solution $35 \mathrm{wt} . \%$ (PAA $\left.M_{w}=15000 \mathrm{~g} / \mathrm{mol}\right)$, ammonia solution $(25 \%)$, nitric acid solution $(2 \mathrm{M})$ were purchased from Sigma Aldrich (France). N,N'-(dimethylphosphonic acid) amino poly(ethylene glycol) $\left(M_{w}=2770 \mathrm{~g} / \mathrm{mol}, 95 \mathrm{wt} . \%\right.$ solution in water, commercial name Optima 100, hereinafter designated by OPT) and poly(sodium methacrylate)-co-poly(oligoethyleneglycol methyl ether methacrylate) (59.3 wt.\% aqueous solution, $M_{w}=52700 \mathrm{~g} / \mathrm{mol}$, commercial name PCP-45, hereinafter designated by PCP) were gifts from Chryso (France). Molecular weight characteristics (weight average mass $M_{w}$, number average mass $M_{n}$, and molar mass dispersity $M_{w} / M_{n}$, all provided by the manufacturer) are resumed in Table 1 . Solutions were made using a Milli-Q water purification system by Millipore.

2.2. Synthesis of akaganeite nanorods. The akaganeite $(\beta-\mathrm{FeOOH})$ nanorods were synthesized using $5.407 \mathrm{~g}$ of $\mathrm{FeCl}_{3} \cdot 6 \mathrm{H}_{2} \mathrm{O}$ dissolved in $100 \mathrm{~mL}$ of Milli-Q water with $0.5 \mathrm{~g}$ of PEI used as a capping agent in a flat-bottomed flask. Magnetic stirring was used to dissolve the iron salt. Then, the solution was heated up to $80^{\circ} \mathrm{C}$ under magnetic stirring $(625 \mathrm{rpm})$ by placing the flask into a heating bath for $2 \mathrm{~h}$ - the time during which a precipitate was formed. The solution $\mathrm{pH}$ was about 1 , as ascribed to hydrolysis of $\mathrm{FeCl}_{3}$ with formation of hydrogen ions. Then, the flask was withdrawn from the heating bath and placed in a water bath at $25^{\circ} \mathrm{C}$. After full cooling the dispersion was centrifuged and washed twice with ethanol for PEI removal.

2.3. Reduction of akaganeite to iron oxide. The wet akaganeite powder was dried at room temperature and then washed once with $\mathrm{NaOH}(1 \mathrm{M})$ to increase the $\mathrm{pH}$ up to 7-8 and twice with MilliQ water using centrifugations between washing steps. This washing allowed obtaining the dispersion at $\mathrm{pH}$ 6-7. The $\mathrm{pH}$ was fixed at nearly neutral value because, as shown by attempts, a basic medium favors polyhedral morphology of iron oxide while an acid medium favors formation of acicular hematite nanoparticles, in accordance with literature ${ }^{32} .2$ $\mathrm{mL}$ of akaganeite neutral $\mathrm{pH}$ dispersion at 0.23 wt. \% was mixed with $25 \mu \mathrm{L}$ of hydrazine aqueous solution (78-82\%), used as a reducing agent, and the dispersion $\mathrm{pH}$ changed to 9-10. The mixture was poured in a glass tube (LabBox, Spain, outer diameter $1 \mathrm{~cm}$, length $10 \mathrm{~cm}$ ) and sealed. The tube was placed in the Microwave Reaction System Microwave 3000 (Anton Paar, Germany) and irradiated by microwaves at a desired power and duration. The use of microwaves allows much more homogeneous heating, so the best control over the reaction. The solution in the tube reached the boiling point $\left(\sim 100^{\circ} \mathrm{C}\right)$ at nearly atmospheric pressure. Once removed from the oven, it was cooled at room temperature and the heating/cooling cycle was repeated for the desired number of times. Akaganeite topotactic transformation into magnetic iron oxide (magnetite) by hydrazine is carried out according to the following equation: $12 \mathrm{BFeOOH}+\mathrm{N}_{2} \mathrm{H}_{4} \rightarrow 4 \mathrm{Fe}_{3} \mathrm{O}_{4}+8 \mathrm{H}_{2} \mathrm{O}+\mathrm{N}_{2}$. Microwave power, duration of heating 
and number of cycles were varied in order to find the optimal set of the parameters allowing the desired nanorod morphology of the synthesized nanoparticles. At the end of the microwave cycles, a dark brown product was obtained and washed once with Milli-Q water, the solution $\mathrm{pH}$ remained unchanged and equal to 9-10.

2.4. Polymer adsorption on the nanorod surface. (a) OPT adsorption at $\mathrm{pH} 6$ (the sample is hereinafter designated by OPT-6). $10 \mathrm{~mL}$ of iron oxide dispersion was mixed with $10 \mathrm{~mL}$ nitric acid solution $(0.25 \mathrm{M})$ and the volume of the dispersion was increased to $70 \mathrm{~mL}$ by adding milli-Q water. The resultant iron oxide dispersion had an iron oxide concentration equal to $1.1 \mathrm{mg} / \mathrm{mL}$, as determined by weighing of the dried powder, and the $\mathrm{pH}$ approximately equal to 6 . This dispersion was divided into seven equal parts each having a volume of $10 \mathrm{~mL}$. Then, each part was sonicated by the ultrasound tip (Ultrasound Bioblock Scientfic Vibra Cell 75041) for $2 \mathrm{~min}$ at the ultrasound power equal to $92 \mathrm{~W}$ by pulses of $5 \mathrm{~s}$ spaced by 2 s "off" periods. Seven different amounts (4.94, 15.8, 31.1, 63.3, 95.0, 127 and $158 \mathrm{mg}$ ) of neat OPT were then added to the iron oxide dispersions, and the mixtures were sonicated by the ultrasound tip for 10 minutes at the same power and period. The $\mathrm{pH}$ upon adding the polymer decreased to 2-3 due to the natural acidity of OPT, which bears two phosphonic acid end-groups.

(b) OPT adsorption at $\mathrm{pH} 1.2$ (the sample is hereinafter designated by OPT-1). $10 \mathrm{~mL}$ of iron oxide dispersion $(0.11 \mathrm{wt} . \%)$ was magnetically decanted on a strong permanent magnet and excess water was eliminated. $20 \mathrm{~mL}$ of nitric acid solution at $0.25 \mathrm{M}$ was then added to the concentrate and the dispersion volume was adjusted to $70 \mathrm{~mL}$ with milli-Q water, while the dispersion $\mathrm{pH}$ was measured at 1.2. The iron oxide dispersion was divided into seven equal parts, treated with ultrasound and mixed with OPT following the same procedure as described in (a). The $\mathrm{pH}$ of all the six dispersions was $\sim 1$.

(c) PCP and PAA adsorption by precipitation-redispersion protocol was realized in the same way as in the case (b), i.e. by setting the $\mathrm{pH}$ value near 1 for the initial iron oxide suspensions and mixing with the quantities of neat PCP or PAA equal to 3.21, 13.1, 26.1, 52.2, 78.3, 104 or $131 \mathrm{mg}$ for PCP and 2.55, 5, 10, 20, 30, 40 or $50 \mathrm{mg}$ for PAA, the mass concentration of iron oxide in each fraction of $10 \mathrm{~mL}$ suspension being kept constant at $1.1 \mathrm{mg} / \mathrm{mL}$. Two ultrasound treatments of the polymer-iron oxide mixture were carried out for $10 \mathrm{~min}$ with setting the solution $\mathrm{pH}$ to 8 by adding $25 \%$ ammonium solution between two treatments. Such method based on a $\mathrm{pH}$ raise from acidic to neutral or weakly alkaline is described in literature as a robust protocol to obtain individually dispersed nanoparticles of oxides, such as cerium oxide $^{33}$ or maghemite ${ }^{34}$ coated by a layer of a weak polyanion like PAA.

To eliminate large aggregates composed of non-stabilized nanorods, all obtained nanorod suspensions were filtered through a filter paper, which caused the concentration of nanorods to decrease from 1.1 to $0.24 \pm 0.02 \mathrm{mg} / \mathrm{mL}$, as measured by Inductively Coupled Plasma Atomic Emission Spectroscopy (ICP-AES).

2.5. Characterization. The synthesized bare akaganeite and iron oxide nanorods, as well as coated iron oxide nanorods were characterized via magnetization and specific area measurements, powder X-ray diffraction (PXRD), Mössbauer spectroscopy, TEM, CryoTEM, DLS, zeta-potential measurements, ICP-AES and thermogravimetric analysis (TGA), as described in detail in SI (Sec. A).

\section{RESULTS AND DISCUSSION}

3.1. Akaganeite and iron oxide nanorods. A TEM micrograph of synthesized akaganeite nanorods (solution $\mathrm{pH} \sim 1$ before washings by ethanol) is shown on Fig. 1a. The histograms of 
the length and diameter distributions obtained from the TEM pictures are shown in Fig. S1a: the nanorods have an average length and diameter equal to 26 and $4 \mathrm{~nm}$, respectively. Recall that PEI was used as a capping agent because it allows an easy control over the size of akaganeite rods and leads to reproducible synthesis of homogeneous nanorods, as shown on Fig. 1a and already reported by Mohapatra et al. ${ }^{4}$ Those authors have shown that varying the PEI amount allows tuning the nanorod length in the range between 30 and $70 \mathrm{~nm}$. The PEI presents polycationic structure and has affinity for a specific crystal faces of akaganeite and therefore favors anisotropic growth of the crystal explaining the final rod-like shape of synthetized nanoparticles. Recently, Lentijo Mozo et al. ${ }^{18}$ have shown that the PEI molecular weight ( 800 vs. $25000 \mathrm{~g} / \mathrm{mol})$ does not influence the length of the akaganeite nanorods, which remains in the range $32-38 \mathrm{~nm}$. The washing step required for the complete PEI removal at the end of the synthesis is fundamental and must be done with an appropriate solvent, such as ethanol. On the contrary, washing with acetone that is not a good solvent for PEI promotes strong aggregation of nanorods during transformation to iron oxide ${ }^{18}$, and does not allow keeping rod-like morphology during transformation of akaganeite to iron oxide.

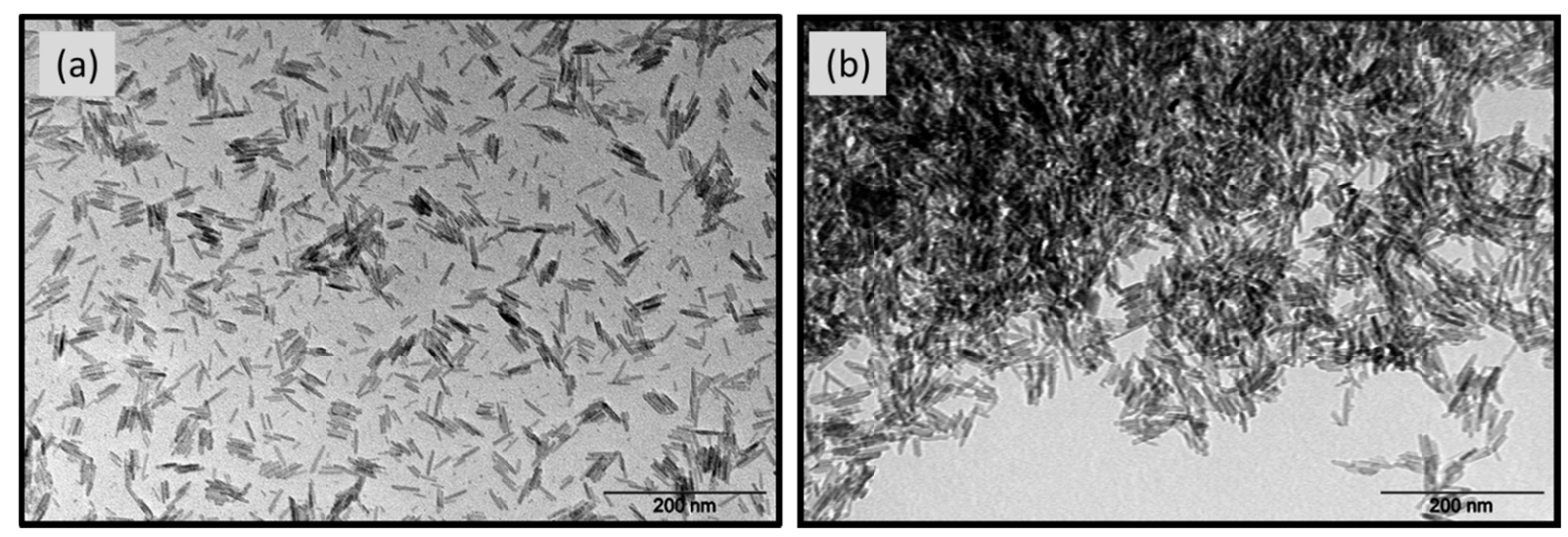

Fig. 1: TEM micrographs of akaganeite nanorods (solution $\mathrm{pH} 1$ before washings by ethanol) (a) and iron oxide nanorods (solution $\mathrm{pH}$ - -10 before drying on the TEM grid) (b). The scale bar is $200 \mathrm{~nm}$.

For transformation of akaganeite to iron oxide, hydrazine is often used as a reducing agent that allows avoiding the appearance of non-magnetic iron oxide ${ }^{20}$. However, preparation of strongly magnetic iron oxide particles requires relatively large amounts of hydrazine at the cost of losing the rod-like morphology. We have checked that increasing hydrazine concentration causes appearance of polyhedral particles mixed with rod-like ones. A reasonable hypothesis for explaining polyhedral shape stands in the "orientation attachment" growth mechanism of nanoparticles that is particularly relevant in oxyhydroxydes ${ }^{35}$ : although akagenite nanoparticles here are synthetic, there exist clay minerals that can stack together, their atomic planes being parallel, and their attachment releases interface tension and decreases the free energy ${ }^{36}$. Therefore, an optimal hydrazine quantity $(\sim 12.8 \mu \mathrm{L} / \mathrm{mL}$ of solution) has been chosen for our synthesis corresponding to the least possible amount of polyhedral particles and to relatively high magnetization. The reaction was carried out in microwave oven to ensure a homogeneous heating, as suggested by Milosevic et al. ${ }^{17}$. The effect of the microwave irradiation parameters on the morphology of synthesized iron oxide nanorods is shown in Table S1. We see that longer periods of microwave exposure $(T=60 \mathrm{~s}$ versus 30 or $45 \mathrm{~s})$, or higher power $(P=300 \mathrm{~W}$ versus $200 \mathrm{~W})$ or larger number of cycles $(n=6$ versus 3 ) give a mixture of rod-like and polyhedral shapes (appearing in TEM images as squares, rectangles, hexagons, etc.) of synthesized nanoparticles (four first rows in Table S1). We have found a set of the microwave parameters ( $T=45 \mathrm{~s}, P=200 \mathrm{~W}$ and $n=3$ ) favoring the 
rod-like shape with a minimum (about a few percent) presence of polyhedral particles (bottom row in Table S1).

The TEM image of iron oxide nanorods (at solution $\mathrm{pH} 9-10$ before drying on the TEM grid) obtained using the aforementioned set of the microwave irradiation parameters is shown on Fig. 1b. The dispersion state of iron oxide nanorods appears to exhibit much more bundles than that of the starting akaganeite nanorods [Fig. 1a]. The length and distribution of individual iron oxide nanorods obtained by image processing is shown on Fig. S1b, while the number average length and diameter are 31 and $6 \mathrm{~nm}$, respectively. Such clustering into dense bundles is not expected from dipolar magnetic interactions between iron oxide nanorods because the dipolar interaction energy remains well below the thermal agitation energy, as shown in SI (Sec. C). The clustering would be favored by the weak alkaline medium caused by the hydrazine addition. During this addition the surface charge of iron oxide nanorods crosses the isoelectric point $\left(\mathrm{pH}_{i e p} \approx 6\right.$, as inferred from zeta-potential measurements, $\mathrm{cf}$. Sec.3.2, black triangles on Fig. 5b) and this likely makes them fall into a deep primary potential minimum (caused by strong van der Waals interactions) such that they are unable to redisperse when $\mathrm{pH}$ rises up to $\sim 9-10$ at the end of hydrazine addition. On the contrary, during akaganeite synthesis, its surface charge does not cross its isoelectric point $\left(\mathrm{pH}_{\text {iep }} \approx 7\right)$ and nanorods are relatively well dispersed at acidic $\mathrm{pH} \sim 1$ at sufficiently large zeta-potential $(\zeta>35$ $\mathrm{mV}$, cf. Sec. 3.2, brown pentagons on Fig. 5b) that is expected to ensure a strong enough electrostatic repulsion.

The specific area of iron oxide nanorods measured by nitrogen adsorption is $S_{B E T}=135$ $\mathrm{m}^{2} / \mathrm{g}$, as compared to the geometric surface $S_{V}=(4 / d+2 / L) / \rho$ equal to $146 \mathrm{~m}^{2} / \mathrm{g}$, with particle density $\rho \approx 5 \mathrm{~g} / \mathrm{cm}^{3}$, average length $L=31 \mathrm{~nm}$ and average diameter $d=6 \mathrm{~nm}$. As we see, the measured area of iron oxide nanorods agrees relatively well with the geometric area likely pointing out to the bulk morphology of nanorods without internal porosity or fusion between individual rods.

The composition and structure of synthesized nanorods were analyzed by PXRD measurements, as well as Mössbauer spectra. Extensive analysis of these characterizations is presented in SI (Sec. B.2, Figs. S2, S3, Table S2) and allows concluding that synthetized iron oxide nanorods contain about $11 \%$ of ordered ferrimagnetic magnetite or maghemite phase and about $89 \%$ of a disordered phase attributed to superparamagnetic magnetite or maghemite mixed with a disordered non-fully transformed akaganeite phase.

The magnetization curve of akaganeite and iron oxide nanorods, i.e. dependency of the nanorod magnetization $M$ (in emu/g) on the magnetic field intensity $H$ (in $\mathrm{kA} / \mathrm{m}$ ) is shown on Fig. 2. Akaganeite nanorods show a weak paramagnetic behavior (dashed red line) at room temperature $(T \approx 300 \mathrm{~K})$, as previously reported for nanorods of near similar size ${ }^{17,37}$ and with Néel temperature (corresponding to antiferromagnetic-paramagnetic transition) about ${ }^{37,38}$ $T_{N}=260 \mathrm{~K}$. The initial volume magnetic susceptibility (estimated as $\chi=\rho(d M / d H)_{H=0}$ assuming the particle density equal to the one of the bulk akaganeite, $\rho \approx 3.5 \mathrm{~g} / \mathrm{cm}^{3}$ ) is about $\chi \approx 10^{-2}$, thus of the same order of magnitude that the one reported by Luna et al. ${ }^{37}$ ( $\chi \approx 6 \times 10^{-3}$, as inferred from the initial slope of the Langevin fit of the magnetization data of that paper).

The synthesized iron oxide nanorods showed a much stronger magnetic behavior (solid curve in Fig. 2). The saturation magnetization $M_{S}$ was estimated as an intercept of a linear extrapolation of the $M$ versus $1 / H$ dependency with the ordinate axis, as suggested by Rosensweig ${ }^{39}$. This gives us the value $M_{S} \approx 13.4 \mathrm{emu} / \mathrm{g}$ (corresponding to $\sim 67 \mathrm{kA} / \mathrm{m}$ assuming the particle mass density equal to the one of bulk iron oxide, $\sim 5 \mathrm{~g} / \mathrm{cm}^{3}$ ), which is about $5-7$ 
times lower than the saturation magnetization of spherical maghemite or magnetite nanoparticles synthesized by a classical co-precipitation route ${ }^{40}$ and usually lower than for bulk iron oxide due to spin canting effects ${ }^{41,42}$. Moreover, the magnetization curve shows a relatively narrow hysteresis loop with remnant magnetization $M_{R} \approx 0.23 M_{S} \approx 3.1 \mathrm{emu} / \mathrm{g}$ (corresponding to $\sim 15.5 \mathrm{kA} / \mathrm{m}$ ) and coercive field $H_{C} \approx 8.9 \mathrm{kA} / \mathrm{m}$. Such magnetic behavior is consistent with nanorod composition $(11 \%$ of ordered ferrimagnetic phase with possible presence of weakly magnetic akaganeite phase) inferred from PXRD and Mösbauer spectroscopy (Sec. B.2 in SI).

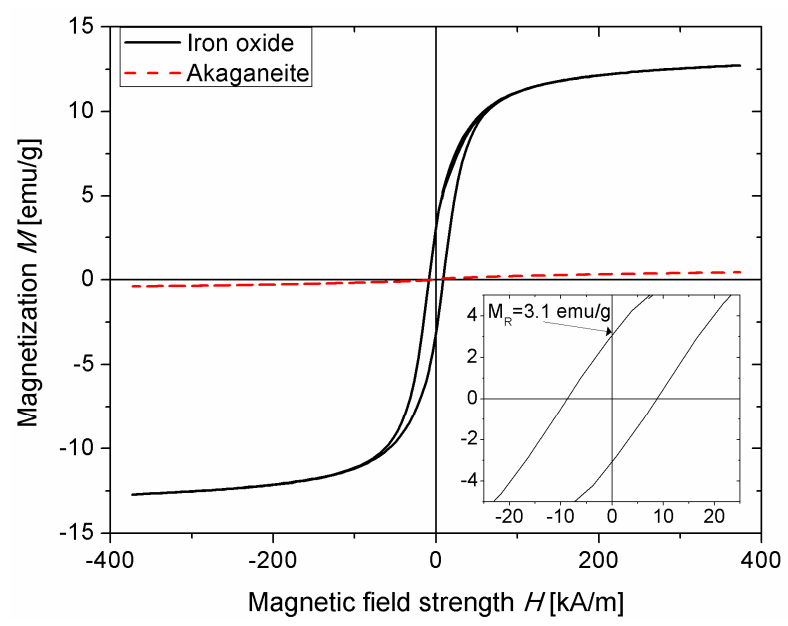

Fig. 2. Magnetization curve of akaganete (dash line) and iron oxide (solid line) nanorods in powder state. The inset shows an enlarged view of iron oxide magnetization curve at small magnetic fields

Existence of magnetic hysteresis confirms the presence of ferro- or ferrimagnetic phase in the nanorod composition. The minimum nanorod volume $V$ above which the nanorods are expected to show ferro- or ferrimagnetic behavior during the typical measuring time $\tau \sim 10 \mathrm{~s}$ is estimated by equating the measuring time with the Néel relaxation time ${ }^{39}$ $\tau_{N}=\tau_{0} \exp \left(K V / k_{B} T\right)$, where $\tau_{0} \sim 10^{-9} \mathrm{~s}$ is a characteristic decay time of magnetic moment precession, $K \sim 3-9 \times 10^{4} \mathrm{~J} / \mathrm{m}^{3}$ is the magnetic anisotropy constant of magnetite ${ }^{43}$ and $k_{B} T \sim 4 \times 10^{-21} \mathrm{~J}$ is the thermal agitation energy at room temperature. This gives us the critical volume of $V \sim 1-3 \times 10^{3} \mathrm{~nm}^{3}$, which is of the same order of magnitude that the geometric nanorod volume $V \sim 0.5-1.5 \times 10^{3} \mathrm{~nm}^{3}$. This hysteresis behavior has been previously reported for nanorods of the average size which is slightly above or nearly the same as the size of our nanorods $(L \times d=50 \times 5 \mathrm{~nm}, 38 \times 12 \mathrm{~nm} \text { and } 32 \times 5.1 \mathrm{~nm})^{5,17,18}$, thus having geometric volume above the critical one. However, smaller nanorods prepared by Orza et al..$^{5}(L \times d=25 \times 5 \mathrm{~nm})$ had the volume below the critical one and showed superparamagnetic behavior without remnant magnetization. Interestingly, Mohapatra et $a l .{ }^{4}$ reports superparamagnetic behavior (or rather, non-discernable coercitivity and remanence) for nanorods in the size range $L \times d=30 \times 3-70 \times 12 \mathrm{~nm}$. However, the blocking temperature below the room temperature (justifying superparamagnetic behavior at room temperature) was only detected for nanorods with the lengths of 30 and $40 \mathrm{~nm}$, which may be due to the expected increase of the anisotropy constant when size decreases, as ascribed to surface contribution to magnetic anisotropy ${ }^{44}$. Concerning saturation magnetization, Orza et $a l .{ }^{5}$ have reported relatively low values of $M_{S} \approx 8 \mathrm{emu} / \mathrm{g}$ for nanorods of small dimension $L \times d=25 \times 5 \mathrm{~nm}$. Milosevic et al. ${ }^{17}$ has explained such low values (also observed for their larger nanorods with $L \times d=38 \times 12 \mathrm{~nm}$ ) by both incomplete transformation of akaganeite to magnetic iron oxide and possible existence of a disordered magnetic layer at the nanorod surface (also called spin-canting). On the other hand, different authors report significant increase of magnetization saturation with the 
nanorod size (from $50 \mathrm{emu} / \mathrm{g}$ at $L=30 \mathrm{~nm}$ to $66 \mathrm{emu} / \mathrm{g}$ at $L=70 \mathrm{~nm}$ and from $8 \mathrm{emu} / \mathrm{g}$ at $L=25$ $\mathrm{nm}$ to $70 \mathrm{emu} / \mathrm{g}$ at $L=50 \mathrm{~nm})^{4,5}$, which probably indicates that formation of ordered magnetic phase is easier for larger nanocrystals, especially if they were grown by an oriented attachment mechanism (thus annealing both crystallographic and magnetic defects).

3.2. Water dispersion of iron oxide nanorods. Three types of polymers were chosen to stabilize the nanorods: biphosphanate ending polyethylene glycol (OPT), sodium polyacrylate (PAA) and sodium polymethacrylate grafted with polyethylene glycol side chains (PCP) (Sec. 2.4). Figure 3 shows a sketch of the iron oxide nanorod coated by these three polymer types. In the case of stabilization by OPT, phosphonate end-groups of the polymer are expected to react with the iron oxide surface, while the PEG chains would extend towards the solvent. Recall that OPT adsorption was realized at two different $\mathrm{pH}$ values of the iron oxide nanorod solution: $\mathrm{pH} \sim 6$ and $\mathrm{pH} \sim 1.2$ (samples OPT-6 and OPT-1). Zeta-potential measurements on bare iron oxide particles [black triangles on Fig. 5b] and ionization curve of OPT [Fig. III-12 in Morini ${ }^{45}$ ] show that at $\mathrm{pH} \sim 1.2$, the particles are positively charged and the phosphonic acid groups of the polymer are almost completely protonated, thus quasi-neutrally charged, while at $\mathrm{pH}=6$, the particles are uncharged (isoelectric point at $\mathrm{pH} \approx 6$ ) and the phosphonate groups are $40 \%$ negatively charged. At the first glance, this is expected to make electrostatic interactions between polymers and particles inefficient. However, it has been reported that the presence of oppositely charged nanoparticles can affect considerably the acid-basic properties of weak polyacids, making the phosphonate group ionization easier at $\mathrm{pH} \sim 1.2^{46,47}$. Although the exact mechanism of OPT adsorption on iron oxide nanorods is not completely clear, isothermal calorimetry measurements ${ }^{48}$ have shown a stronger bonding energy of phosphonates compared to other ligands like sulfonic or carboxylic acids, as also found by other authors who compared the efficiency of phosphonate-terminated PEG to carboxylate or amine as anchoring moieties on the iron oxide surface ${ }^{49}$. We can even deduce that phosphonates do not only interact with the oxygen atoms of the oxide surface through dipolar forces or hydrogen-bonds, but also directly with the iron atoms, in their "inner sphere" (fist layer of hexa-coordinated ligands), by coordination bonds that are intermediate between dipolar and covalent bonds ${ }^{50}$.

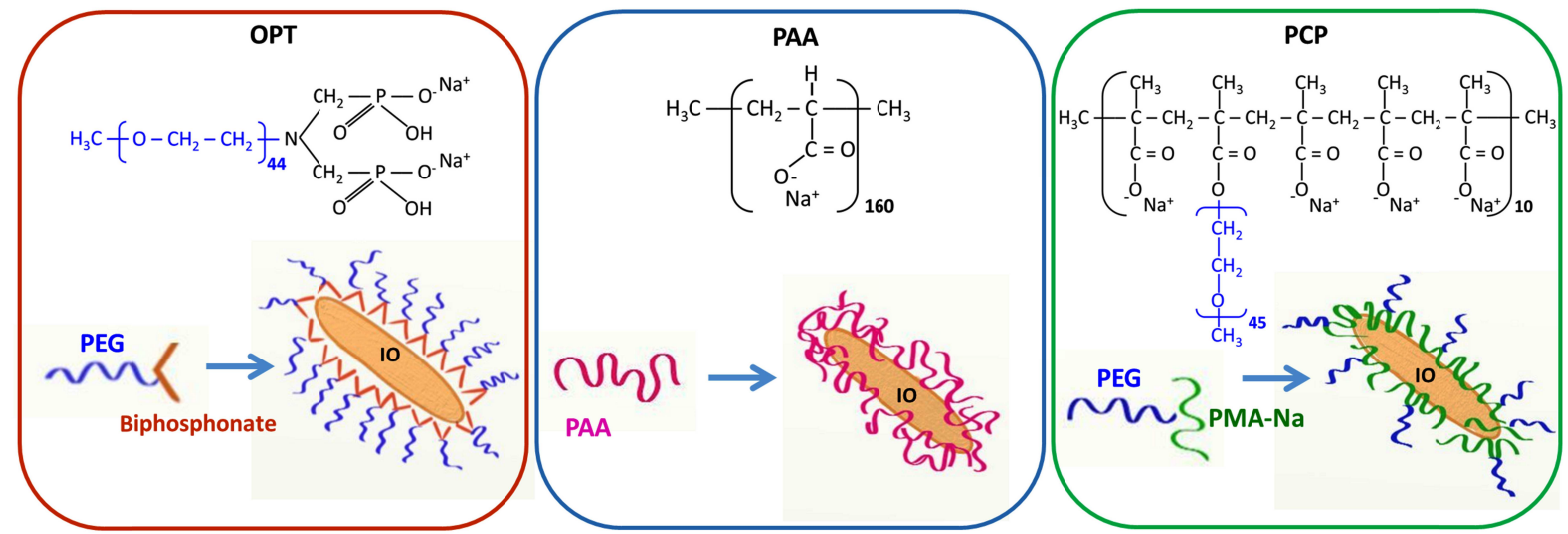

Fig. 3. Schematic representation of an iron oxide (IO) nanorod coated with OPT, PAA and PCP

In the case of PAA and PCP molecules which bear multiple carboxylate moieties as adsorption points, a well-known precipitation-redispersion protocol was used to stabilize the nanorods. This method consists in making the complex between the polymer (polyelectrolyte) and the iron oxide at very acid $\mathrm{pH}(\sim 1.2)$ followed by redispersion of particles covered with the polymer at $\mathrm{pH}>8$, which allows extension of polymer chains ${ }^{33,34}$. The PAA and PCP molecules are expected to bind by multiple carboxylic groups to the iron oxide surface of the nanorods (although carboxylates are much weaker ligands than phosphonates, their 
multivalence favors the adsorption). The PEG side chains of the PCP molecules extending into the solvent and unbound "loops" of PAA molecules bring steric repulsion and the negative charge at basic $\mathrm{pH}$ of the unbound carboxylates of PCP and PAA are expected to bring electrostatic repulsion between nanoparticles, which is however screened by high solution ionic strength (Debye length $\kappa^{-1} \approx 0.9 \mathrm{~nm}$ ), as proved by estimates in SI (Sec. C).

Before studying the colloidal stability of the coated nanorods, we should emphasis that magnetostatic interactions alone (due to remnant nanorod magnetization $M_{R} \sim 15.5 \mathrm{kA} / \mathrm{m}$ ) cannot lead to the inter-particle aggregation at the considered nanorod size, as shown by estimates in SI (Sec. C). The main reason for possible aggregation should rather come from van der Waals interactions in case of inefficient polymer coating.

(a)

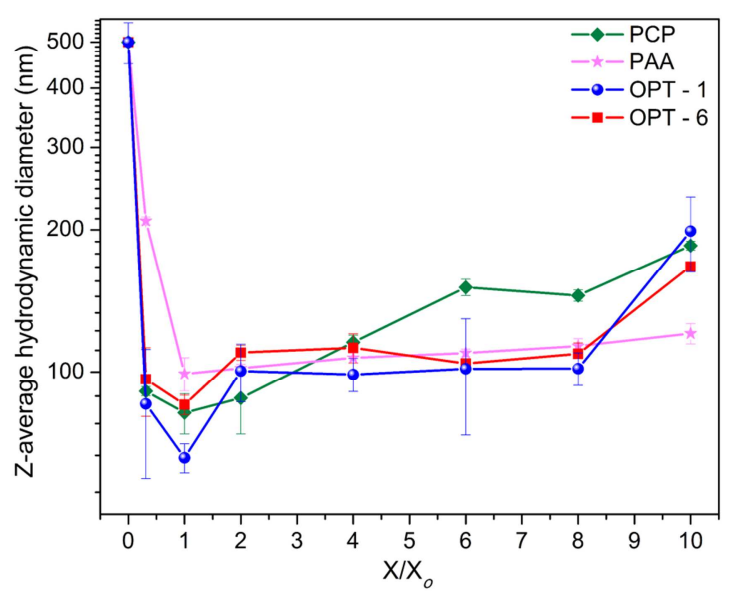

(b)

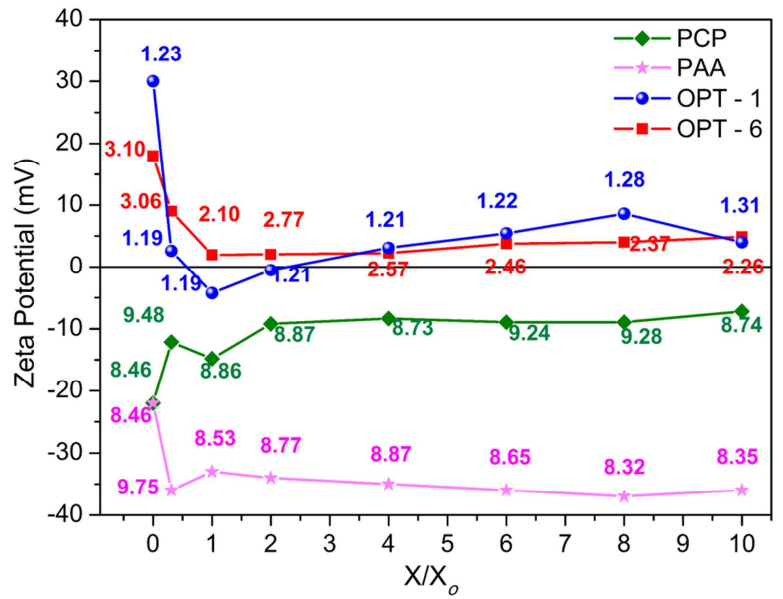

Fig. 4. $Z$-average hydrodynamic diameter (a) and zeta potential (b) as a function of $X / X_{o}$, where $X$ is the ratio of the mass concentration of the added polymer to the mass concentration of nanorods in the suspension and $X_{o}$ is the optimal mass ratio corresponding to minimal hydrodynamic size of nanorods. $X_{o}=0.5,1.3 \mathrm{and} 1.6 \mathrm{mg} / \mathrm{mg}$ for PAA, PCP and OPT, respectively (cf. Table 1). The numerical values indicated above each experimental point in (b) are the initial values of $\mathrm{pH}$ after preparing the suspensions. The same $\mathrm{pH}$ values apply to the points in (a).

The $Z$-average hydrodynamic effective diameter, $d_{Z}$, of nanorods directly assessed from DLS measurements has been chosen as a rapid indicator of the nanorod suspension stability. The effective size larger than geometrical dimension of an individual nanorod is a signature of nanorod aggregation, as individually dispersed nanorods theoretically exhibit an equivalent hydrodynamic diameter smaller than their length. Before analyzing absolute values of the average particle size, let us inspect qualitative effects of the polymer quantity and of the $\mathrm{pH}$ on the suspension stability [Figs. $4 \mathrm{a}$ and 5a, respectively, also see Table S3 in SI]. The polymer amount added to the suspension was characterized by the ratio $X$ of the mass of the added polymer molecules to the mass of iron oxide nanorods. The value of $X$ was calculated as the ratio $X=c_{w p} / c_{w N R}$ of the mass concentration $c_{w p}$ of the added polymer (in $\mathrm{mg} / \mathrm{mL}$ ) to the mass concentration of the nanorods in the suspension $c_{w N R}=1.1 \mathrm{mg} / \mathrm{mL}$ before filtration of the samples. Note that after filtration, the nanorod concentration decreased to $c_{w N R}=0.24 \pm 0.02$ $\mathrm{mg} / \mathrm{mL}$, as estimated by ICP-AES measurement [SI, Sec. A]. The dependency of the Zaverage particle size, $d_{Z}$, as function of the mass ratio $X$ is shown in Fig. 4a. The $\mathrm{pH}$ of these suspensions was not changed and was approximately equal to the $\mathrm{pH}$ at the end of the dispersion of nanoparticles [Sec. 2-4], called hereinafter initial $\mathrm{pH}(\approx 9$ for PCP and PAA samples, $\approx 1.2$ and $\approx 3$ for OPT-1 and OPT- 6 samples, respectively, independently of $X$ ). All the curves for four samples exhibit a minimum of the $d_{Z}(X)$ dependency at an optimal value of $X_{o}$ equal to $0.5,1.3$ and $1.6 \mathrm{mg} / \mathrm{mg}$ for PAA, PCP and OPT molecules, respectively. These values of $X_{o}$ are resumed in Table 1. Zeta potential [Fig. 4b] and electrical conductivity measurements [Table S3] for all the samples as function of the polymer amount show that the 
nanoparticle effective charge do not evolve significantly with $X$ at $X \geq X_{o}$ for all the samples [Fig. 4b] at their initial $\mathrm{pH}$ (indicated above each point in Fig. 4b). This means that the intensity of electrostatic interactions between nanoparticles does not show important changes with $X$ at $X \geq X_{o}$ and cannot explain the increasing part of the $d_{Z}(X)$ dependency. The following scenario explaining the minimum of $d_{Z}(X)$ dependency is proposed.

Below $X_{o}$, the nanorod surface is expected to be only partially covered by polymer molecules. Therefore, as the ratio $X$ increases, the polymer amount on the particle surface should progressively increase until saturation. A progressive change of the particle zeta potential for four samples up to a plateau value at $X>X_{o}$ [Fig. 4b] correlates with progressive polymer adsorption with increasing $X$. As the steric repulsion between nanorods increases with the surface density of adsorbed molecules, the nanorod colloidal stability is expected to be progressively improved with initial increase in $X$. This explains the initial decrease of effective particle size by increasing $X$. Once the particle surface is saturated, further addition of polymers to the nanorod dispersion will increase the amount of non-adsorbed polymers, present as soluble chains in the medium, potentially forming a deleting layer around the nanorods. This increases their osmotic pressure and can generate attractive depletion interaction between nanorods when non-adsorbed polymers are squeezed out form the gap between closely spaced particles that creates an asymmetric osmotic pressure distribution over the nanorod surface pushing two nanorods towards each other ${ }^{51}$. This likely explains an increase of the effective particle size with $X$ at $X>X_{o}$. At least, theoretical estimations in Sec. 3-3 support this effect.

Because of presumably small polymer quantities adsorbed on the iron oxide nanorods at $X<X_{o}$, both ICP-AES and TG analyses were unable to measure with confidence the amount $\mathrm{X}_{a d s}$ of adsorbed molecules per unit particle mass. However, at $X \geq X_{o}$ the ICP-AES analysis showed that the amount of adsorbed OPT molecules was, within the experimental error, independent of the amount $X$ of added polymer, and equal to $X_{a d s}=1.5 \pm 0.5 \mathrm{mg} / \mathrm{mg}$, which supports the aforementioned hypothesis on saturation of the iron oxide surface at $X \geq X_{o}$, at least for the OPT samples (see adsorption isotherm on Fig. S4 and associated discussion in SI). More precisely, the difference in $X_{a d s}$ was undistinguishable from the error bars of the ICP-AES analysis for both OPT-1 and OPT-6 samples, supporting saturation. For the two other polymers less easy to titrate because they do not contain phosphorus, TGA on PCP samples gave a rough estimate of the amount of adsorbed PCP molecules $X_{a d s}=0.15 \pm 0.05$ $\mathrm{mg} / \mathrm{mg}$, while the TGA on PAA samples did not allow distinguishing between adsorbed and non-adsorbed polymers.

Reported values of $X_{a d s}$ allow estimation of the surface density of the polymer chains on the nanorod surface using the following relationship: $n=X_{a d s} N_{A} /\left(M_{n s} S_{B E T}\right)$, where $N_{A} \approx 6 \times 10^{23} \mathrm{~mol}^{-1}$ is the Avogadro constant, $M_{n s}$ is the number average molecular weight of the adsorbed polymer segment $(2550 \mathrm{~g} / \mathrm{mol}$ for OPT and $2100 \mathrm{~g} / \mathrm{mol}$ for 1 methacrylate segment of PCP bearing one PEG side chain) and $S_{B E T} \approx 135 \mathrm{~m}^{2} / \mathrm{g}$ is the specific area of nanorods measured by nitrogen adsorption [Sec. 3-1]. The values of the grafting density of PCP and OPT polymers are resumed in Table 1. The surface density of PEG chains of OPT and PCP molecules is estimated to be of the order of $n \approx 3 \pm 1$ and $0.3 \pm 0.1 \mathrm{~nm}^{-2}$, respectively. The value $n \approx 3 \pm 1 \mathrm{~nm}^{-2}$ (as inferred from adsorption isotherm in Fig. S4) for bisphosphonatePEG molecules (OPT sample) in our study is the same as that reported by Miles et $a l^{49}$ for PEG of 2900 or $3400 \mathrm{~g} / \mathrm{mol}$ grafted onto iron oxide nanopartciles of a diameter of $8 \mathrm{~nm}$ and approaches a maximum grafting density $n_{\max } \approx 3.5 \mathrm{~nm}^{-2}$ for fully stretched mono-phosphonatePEG molecules ${ }^{52}$. Our value $\left(n \approx 3 \pm 1 \mathrm{~nm}^{-2}\right)$ is somewhat higher than the one $\left(n \approx 1 \mathrm{~nm}^{-2}\right)$ measured by Qi et al. ${ }^{52}$ and Torrisi et al. ${ }^{23}$ for comb-like phosphonate-PEG polymers adsorption onto cerium oxide and maghemite spherical nanoparticles, respectively. A higher 
grafting density of OPT molecules on our nanorods likely comes from a stronger binding of the bi-phosphonate-PEG molecules to the iron oxide surface as compared to monophosphonate-PEG molecules in the study of Qi et al. ${ }^{52}$ or Richard et al. ${ }^{53}$ who reported grafting densities up to $2.3 \mathrm{~nm}^{-2}$ on spherical iron oxide nanoparticles, and those obtained with a grafted architecture by Torrisi et al. ${ }^{23}$. However the surface area of a phosphonate head-group being $0.24 \mathrm{~nm}^{2}$, the value above $3 \mathrm{~nm}^{-2}$ found in the present study can still be compatible with a monolayer ${ }^{54}$. The grafting density $n \approx 0.3 \pm 0.1 \mathrm{~nm}^{-2}$ of the PEG chains of PCP molecules onto the iron oxide surface in the present study is close to that $\left(\sim 0.2 \mathrm{~nm}^{-2}\right)$ for PCP molecules adsorbed on calcium carbonate surface ${ }^{45}$ and is much smaller that the grafting density of OPT molecules likely because of geometrical constrains imposing a larger minimum distance between the anchoring points of PEG chains (separated by five methacrylate groups) of PCP molecules as compared to OPT molecules.

Table 1. Main characteristics of polymers and main results on polymer adsorption/nanorod stabilization

\begin{tabular}{|c|c|c|c|c|c|c|c|c|}
\hline \multirow[t]{2}{*}{ Polymer } & \multicolumn{3}{|c|}{ Molecular weight } & \multirow[t]{2}{*}{$\begin{array}{l}\text { Grafting } \\
\text { density } \\
n, \mathrm{~nm}^{-2}\end{array}$} & \multirow{2}{*}{$\begin{array}{c}\text { Estimated } \\
\text { adsorbed } \\
\text { layer } \\
\text { thickness } \\
\delta[\mathrm{nm}]\end{array}$} & \multirow{2}{*}{$\begin{array}{c}\text { Optimal } \\
\text { amount of } \\
\text { added } \\
\text { polymer } X_{O} \\
{[\mathrm{mg} / \mathrm{mg} \text { part }]}\end{array}$} & \multicolumn{2}{|c|}{$\begin{array}{l}\text { DLS hydrodynamic } \\
\text { size of coated } \\
\text { nanorods }\end{array}$} \\
\hline & $\begin{array}{l}M_{w} \\
{[\mathrm{~g} / \mathrm{mol}]}\end{array}$ & $\begin{array}{l}M_{n} \\
{[\mathrm{~g} / \mathrm{mol}]}\end{array}$ & $M_{w} / M_{n}$ & & & & $d_{Z}[\mathrm{~nm}]$ & $P D I$ \\
\hline OPT-1 & 2770 & 2550 & $\sim 1.1$ & $3 \pm 1$ & $6.9 \pm 0.8$ & 1.6 & 65 & 0.15 \\
\hline OPT-6 & 2770 & 2550 & $\sim 1.1$ & $3 \pm 1$ & $6.9 \pm 0.8$ & 1.6 & 84 & 0.16 \\
\hline PCP & 52700 & 21000 & $\sim 2.5$ & $0.3 \pm 0.1$ & $3.3 \pm 0.4$ & 1.3 & 82 & 0.27 \\
\hline PAA & 15000 & 10700 & $\sim 1.4$ & N/A & $\mathrm{N} / \mathrm{A}$ & 0.5 & 99 & 0.16 \\
\hline
\end{tabular}

The effects of solution $\mathrm{pH}$ on average effective particle size and on the particle zeta potential at the optimal concentration $X=X_{o}$ of added polymer are inspected in Fig. 5a and 5b for four samples. The solution $\mathrm{pH}$ does not considerably influence the dispersion state of both OPT samples, which is expected because the conformation of the neutral PEG chains of the OPT molecule are unaltered by $\mathrm{pH}$ while the effective charge of the particle remains negligible at any $\mathrm{pH}$ (the absolute value of the zeta potential remains lower than $10 \mathrm{mV}$ ), such that electrostatic interactions between particles are negligible for any $\mathrm{pH}$ in the considered range, and the particle stability is fully ensured by steric repulsion, as shown by theoretical estimates in Sec. 3-3. On the contrary, the average effective size of nanorods covered with PAA and PCP molecules significantly increases with decreasing $\mathrm{pH}$, as previously observed for PAA complexes with cerium oxide ${ }^{33}$ and with iron oxide ${ }^{34}$. This could be explained by a combination of the two following effects, the first of which is discussed for the PAA molecule, since the polymethacrylate skeleton of the PCP molecule is expected to show qualitatively similar behavior: (a) Conformation of PAA chains adsorbed onto iron oxide nanorods should depend on both the anchoring density and on interactions with the solvent. Using the precipitation-redispersion protocol, PAA chains are expected to be adsorbed to nanorod surface by several monomers (trains) [as estimated by Sehgal et al. ${ }^{33}$ ], while the rest of the monomers point out towards the solvent in a form of loops or tails. When dispersed in the solvent, PAA chains exhibit coil-to-globule transition at $\mathrm{pH} \approx 5$ that is close to the $\mathrm{pKa}$ value of carboxylates ${ }^{55}$. When being adsorbed at a solid surface, polymers likely adopt "pancake-like" conformation at low $\mathrm{pH}$ with the thickness well below the gyration radius in the solvent. With increasing $\mathrm{pH}$, the PAA ionization degree increases and the loops and tails of the polymer are expected to be more stretched towards the solvent, thus increasing the effective thickness of the adsorbed layer and decreasing attractive van der Waals interactions between the nanorods. This mechanism is schematically illustrated in Fig. 5c. (b). Because of the low ionization degree of PAA and PCP molecules at acidic $\mathrm{pH}$ (about $3 \%$ for PAA and $5 \%$ for $\mathrm{PCP}$ at $\mathrm{pH}=4$ as inferred from dissociation curves in Swift et al. ${ }^{55}$ and Morini ${ }^{45}$ ), the particle charge (or equivalently, the zeta potential) is very low below $\mathrm{pH}=4$ [Fig. 5b]. This 
should give a low electrostatic repulsion barrier between nanorods at acidic $\mathrm{pH}$, while electrostatic repulsion is expected to increase at basic $\mathrm{pH}$ with an increasingly more negative effective particle charge [Fig. 5b]. Nevertheless, we have to exclude scenario (b) because, as mentioned above, the range of the electrostatic repulsions is found to be very short at any solution $\mathrm{pH}$ (Debye length, $\kappa^{-1} \approx 0.9 \mathrm{~nm}$, see SI, Sec. C).

Notice that the effective particle charge is nearly zero at $\mathrm{pH}<4$ for all four samples stabilized by different molecules at optimal mass ratio $X=X_{o}$ [Fig. 5b], while uncovered particles bear significant positive charge $(\zeta \sim+30 \mathrm{mV}$ at $\mathrm{pH}=2)$. Since all the three polymers are very weakly charged at $\mathrm{pH}<4$, the neutral charge of covered particles means that all the $\mathrm{Fe}-\mathrm{OH}$ sites of the iron oxide surface are linked to the phosphonic or carboxylic groups of the polymers, which correlates with the saturation of the nanorod surface with adsorbed molecules supposed at $X \geq X_{o}$. On the other hand, the ionization degree of the free PAA and PCP molecules tends to unity at $\mathrm{pH}>10$, such that we would expect a maximum particle charge at $\mathrm{pH}>10$, as observed in Fig. 5b. Numerical estimation shows that at $\mathrm{pH}=10$, the particle charge density represents only $0.6 \%$ for PAA and $2 \%$ for PCP of the maximum charge that the particles would possess if all the carboxylic groups of adsorbed PAA and PCP molecules were fully ionized. Such low charge density values cannot be explained by the fact that the most of the carboxylic groups are adsorbed onto the nanorod surface canceling the polyelectrolyte charge because this would imply unrealistically high density of the iron oxide - carboxylate bonds (up to 24 bonds per $\mathrm{nm}^{2}$ for PAA and 4 bonds per $\mathrm{nm}^{2}$ for PCP). As already stated, the polyelectrolyte shell on the nanorod surface is expected to show a sequence of loops and trains, as shown schematically on Fig. 3 and 5c. At this configuration, counterions could penetrate into the polyelectrolyte shell and strongly decrease the overall effective particle charge measured through particle electrophoretic mobility (or zeta potential), as has been found theoretically and experimentally ${ }^{56-58}$.
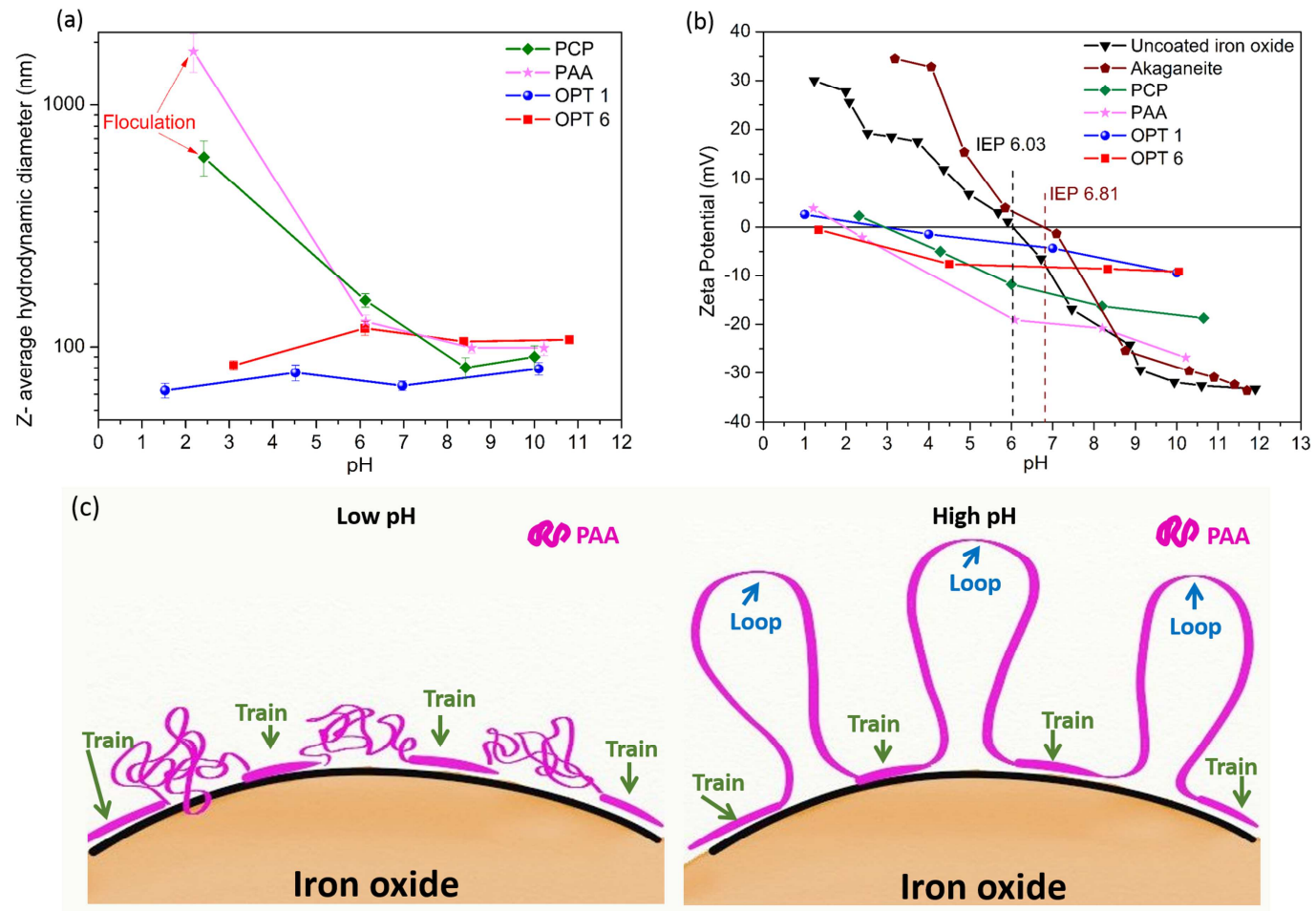

Fig 5. Z-average hydrodynamic diameter (a) and zeta potential (b) as a function of solution $\mathrm{pH}$ at the mass ratio $X=X_{o}(=0.5,1.3$ and $1.6 \mathrm{mg} / \mathrm{mg}$ for PAA, PCP and OPT, respectively, see Table 1); a schematic representation of possible PAA conformation on the particle surface as function of $\mathrm{pH}$ is shown in (c) 
The typical size distributions of particles at the optimal conditions (at $X=X_{o}$ and at basic $\mathrm{pH}$ for PAA and PCP) are shown on the upper row of Fig. 6 for the four considered samples, while the TEM micrographs of the same samples are shown on the bottom row of this figure. The values of the average hydrodynamic particle size, $d_{Z}$, and of the DLS polydispersity index $P D I$ are resumed in Table 1 . Over the whole studied range of $\mathrm{pH}$ and of polymer concentration, the lowest effective particle size $\left(d_{Z} \approx 65 \mathrm{~nm}\right)$ at a relatively low value of polydispersity index, $P D I \approx 0.15$, so the best dispersion state, was observed for samples stabilized with OPT-1 method, while the samples stabilized by PAA gave the largest particle size $\left(d_{Z} \approx 99 \mathrm{~nm}\right)$ but still single peak distribution with a good $P D I \approx 0.16$, and the PCP samples gave double peak distribution with intermediate average size $d_{Z} \approx 82 \mathrm{~nm}$ and a relatively high $P D I \approx 0.27$. Thus, PAA and PCP were judged to be less efficient for nanorod stabilization than OPT. The TEM micrographs of Fig. 6 confirm a better dispersion state of the OPT-1 samples as compared to PCP and PAA ones, all the four samples being at the optimal mass ratio $X=X_{o}$. However, we still observe lateral stacking of nanorods even for the best dispersion state of the OPT-1 sample.
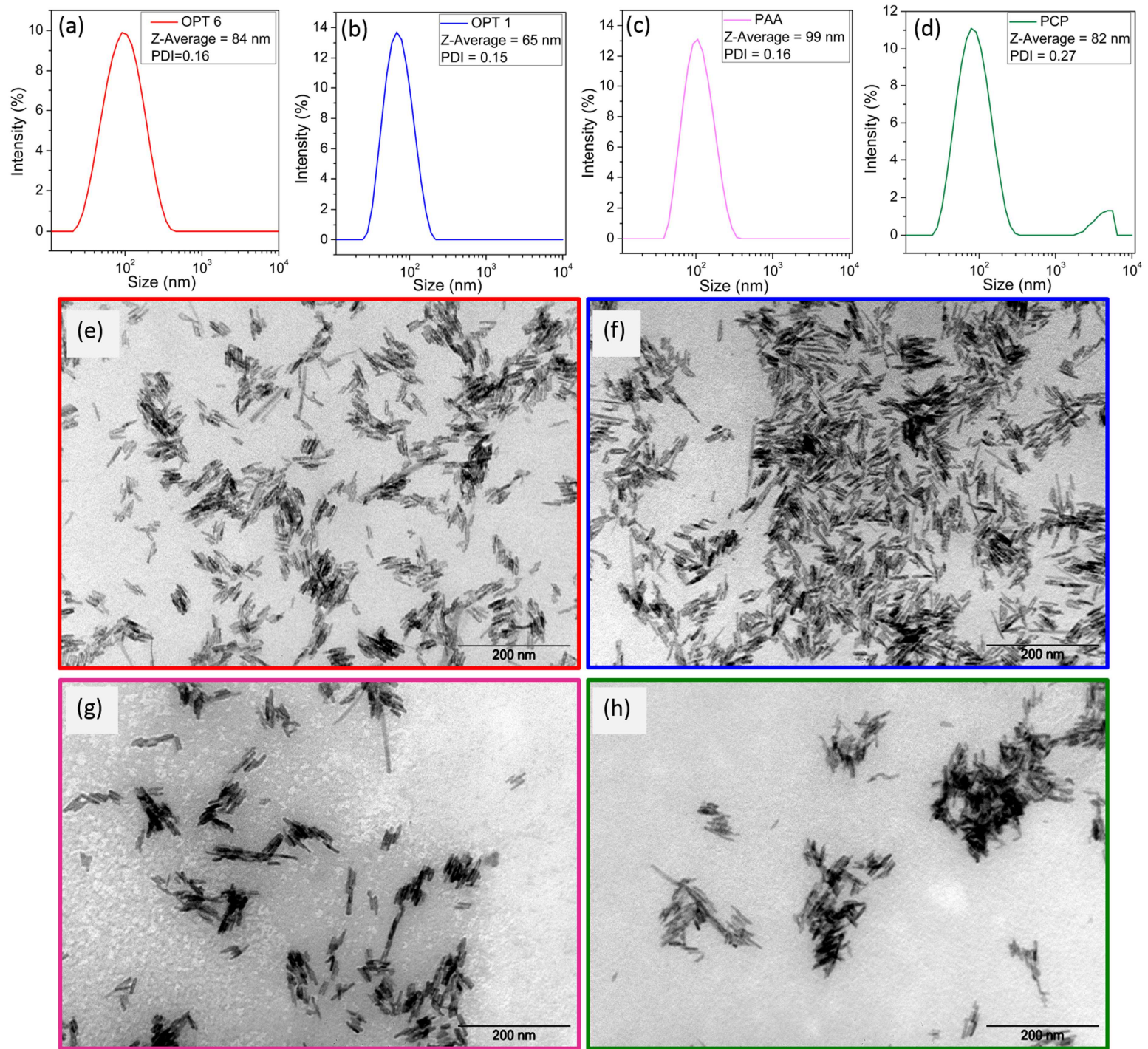

(h)

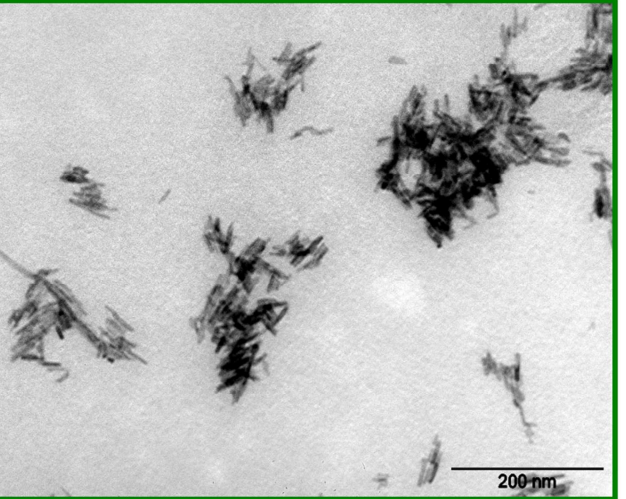

Fig. 6. Intensity size distribution measured by DLS and TEM micrographs of iron oxide nanorods coated with different molecules OPT-6 (a,e), OPT-1 (b,f), PAA (c,g), PCP (d,h). The scale bar in (e) - (h) is $200 \mathrm{~nm}$.

As aggregation might be induced during drying of the liquid sample on the grid before inserting it into the electronic microscope, we performed cryo-TEM experiments to check this point. In that case the sample was rapidly frozen, which keeps its initial dispersion state 
unaffected by drying. In figure S5, we compare TEM and Cryo-TEM micrographs for the sample OPT- 6 at optimal amount of added polymer $X=X_{o}$ and at the same initial concentration of particles in the liquid solvent. Smaller aggregates are observed in the Cryo-TEM micrograph, corroborating the hypothesis that the aggregation is to some extent induced by drying the sample on the TEM grid. However, Cryo-TEM does not show ideal nanorod dispersion, as some aggregates consisting of a few laterally stacked nanorods are still observed. Note that the Z-average size $d_{Z}$ of nanorods above $60 \mathrm{~nm}$ (Fig. 6a, b) corroborate with existence of aggregates because $d_{Z}$ refers to a diameter of an equivalent sphere whose diffusion coefficient is equal to the average (over all possible orientations) diffusion coefficient of nanorods. Thus, evaluation of the diffusion coefficients (Sec. B-4 in SI) shows that if the nanorods were ideally dispersed, their Z-average diameter would be about 2 times smaller than their TEM intensity averaged length $L_{T E M, I} \approx 40 \mathrm{~nm}$ giving $d_{Z} \approx 20 \mathrm{~nm}$ instead of $\sim 60 \mathrm{~nm}$.

3.3. Evaluation of the colloidal stability. At this point, we attempt to explain the existence of aggregates and the difference in aggregation state of different samples, as well as the effect of the polymer mass ratio $X$, by theoretical estimates of the energy of interaction between two parallel nanorods of a length $L$ and diameter $d$ as function of the distance $h$ separating their lateral metal oxide surfaces. According to estimates (SI, Sec. C), the magnetostatic interaction is negligible. Electrostatic interactions between nanorods covered with PAA or PCP are very short ranged (Debye length $\kappa^{-1} \approx 0.9 \mathrm{~nm}$ ), while the nanorods coated with OPT have a weak zeta potential [Fig. 5b] making electrostatic inetearction negligible for all polymers. In the case of the optimal mass ratio $X=X_{o}$, the amount of non-adsorbed polymer is expected to be low enough and to lead to negligible depletion interaction. However, depletion interaction cannot be ignored for higher polymer concentrations. Thus, the main interactions defining nanorod dispersion state are van der Waals attraction, steric repulsion and depletion attraction, while the total interaction energy $U$ is simply the sum of the energies of these three interactions, which are evaluated in SI (Sec. C). Similar evaluations have been presented in SI of the paper by Schrittwieser et al. ${ }^{12}$, however depletion and steric interactions, relevant for our system, were not taken into account.

Theoretical dependencies of the total interaction energy $U$ on the separation $h$ between nanorod metal oxide surfaces are plotted on Fig. 7a and 7b for OPT and PCP molecules, respectively for three different concentrations $c_{w}$ of free polymer in the suspending fluid (found by ICP-AES analysis for the OPT sample - cf. SI, Sec. A) and corresponding to three values of the added polymer amount $X \approx X_{o}, 4 X_{o}$ and $10 X_{o}$, where $X_{o}$ values are presented in Table 1 for each polymer. Since the amount of polymer adsorbed onto the nanoparticle surface was determined with a large error, all the $U(h)$-curves are plotted for two values of the grafting density $n$, corresponding to the lower and the upper bounds of the experimental interval of $n$ values.

Let us firstly inspect the behavior of OPT samples. As inferred from Fig. 7a, the theory predicts a strong repulsive barrier for OPT samples in the considered grafting density interval $n=2-4 \mathrm{~nm}^{-2}$ and at all considered concentrations $c_{w}=1-14 \mathrm{mg} / \mathrm{mL}$ of the free polymer (denoted in as $c_{w, O P T_{-} \text {free }}$ in SI, Sec. A). However, with a decrease of the grafting density the repulsive barrier shifts to smaller interparticle distance $h$. Since we do not have data on the amount $X_{a d s}$ of adsorbed OPT polymer for $c_{w}<1 \mathrm{mg} / \mathrm{mL}$ (or $X<X_{o}$ ), we can qualitatively inspect the colloidal stability of nanorods at some arbitrary value of the grafting density beyond the measured interval. For example, at $n=0.3 \mathrm{~nm}^{-2}$ and $c_{w}$ taken to be equal to 0 (negligible concentration of free polymer), the total interaction energy is negative at all $h$ and its absolute value becomes well below the thermal agitation energy $k_{B} T$ at $h<1 \mathrm{~nm}$ (see the dashed line on Fig. 7a), indicating that the particles undergo irreversible aggregation. 
Therefore, progressive increase of the grafting density from $n=0$ (bare particles) to $n=2-4 \mathrm{~nm}^{-2}$ (particle surface saturated with OPT) leads to an increase of the repulsive barrier energy and shifts this barrier to higher separations $h$, which improves the nanorod colloidal stability - the effect corroborating with an initial decrease of the average nanorod size with increasing $X$ values [Fig. 4a]. It is remarkable that, at maximum grafting density $n=2-4 \mathrm{~nm}^{-2}$, the theoretical $U(h)$ dependencies for OPT samples exhibit the secondary energy minima, whose depth progressively increases from $-1 k_{B} T$ to $-11 k_{B} T$ as the free polymer concentration increases from $c_{w}=1 \mathrm{mg} / \mathrm{mL}$ to $14 \mathrm{mg} / \mathrm{mL}$. This is explained by an increase of the attractive depletion potential with an increase of $c_{w}$ and agrees with the increase of the average nanorod hydrodynamic size with increasing $X$ at $X>X_{o}$ [Fig. 4a]. Since the steric repulsion energy diverges as $U_{s t} \sim+h^{-3 / 4}$ [Eq. (S-7)] and the van der Waals attractive energy diverges as $U_{v d W}$ $\sim-h^{-3 / 2}$ [Eq. (S-6)] at $h \rightarrow 0$, the resultant interaction is attractive in the vicinity of close contact between metal oxide surfaces of opposite nanorods, which points out to existence of a primary energy minimum for OPT samples. However, at maximum grafting density $n=2-4$ $\mathrm{nm}^{-2}$, the primary minimum is situated at unreally small distances $h<<1 \mathrm{~nm}$, such that in practice it does not appear.
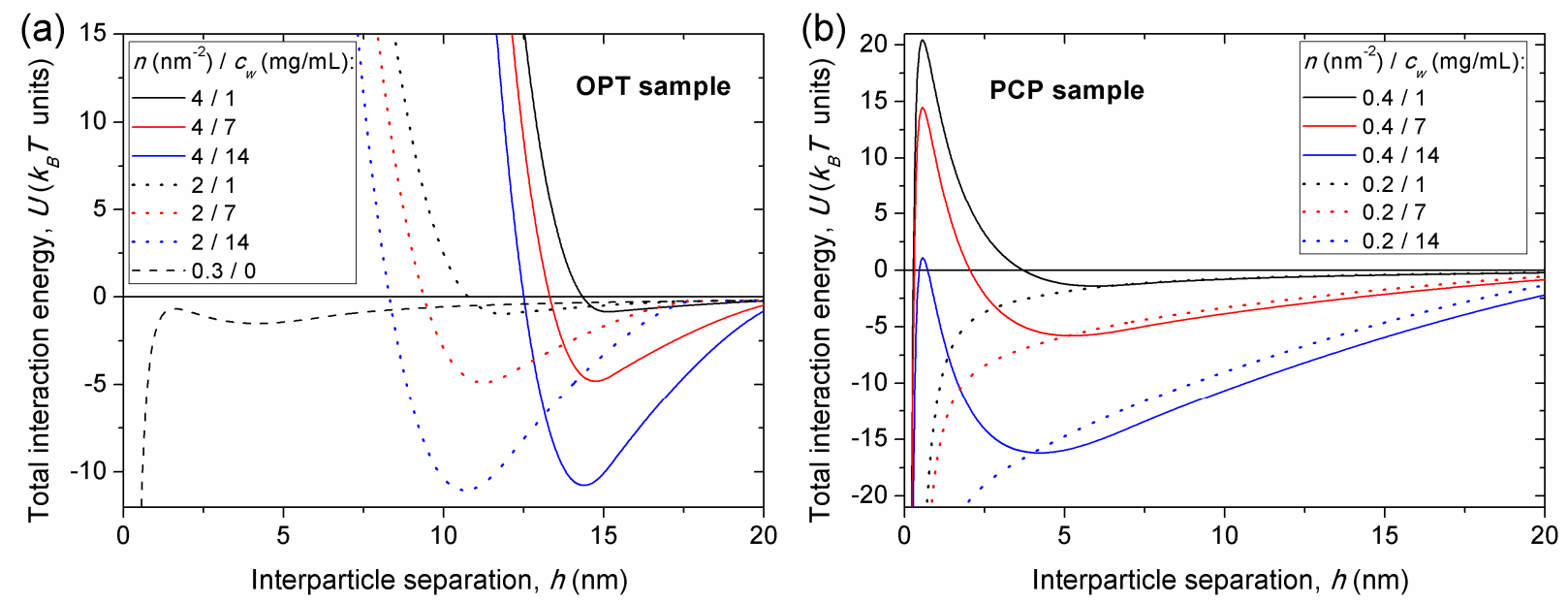

Fig. 7. Theoretical dependency of the total energy of interaction between parallel iron oxide nanorods covered with a polymer brush on the lateral separation between nanorods for two different grafted molecules: OPT (a) and PCP (b). The calculations were done for three different concentrations of the free polymers, as well as the lower and the upper bounds of measured grafting densities for each grafted molecule $\left(n=2-4 \mathrm{~nm}^{-2}\right.$ for OPT and $0.2-0.4 \mathrm{~nm}^{-2}$ for PCP). In addition to these values of $n$, the dashed black line in (a) corresponds to $n=0.3 \mathrm{~nm}^{-2}$ for the OPT sample.

Since the difference between the measured values $X_{a d s}$ of OPT-1 and OPT-6 samples is undistinguishable from relatively large experimental errors, we cannot conclude with confidence that better dispersion state of OPT-1 samples comes from higher grafting density as compared to the one for OPT-6 samples. For both OPT samples, at the optimal concentration of the free polymer $c_{w} \approx 1 \mathrm{mg} / \mathrm{mL}$ (corresponding to $X=X_{o}=1.6 \mathrm{mg} / \mathrm{mg}$ ), the nanorods are expected to be stable against aggregation since the energy minimum $\left(-1 k_{B} T\right)$ is of the order of magnitude of the thermal agitation energy. However in experiments, we still observe some aggregation even at optimal polymer concentration, as evidenced by Cryo-TEM [Fig. S5]. Such discrepancy could come from the fact that performed theoretical estimations likely overestimate the repulsive interactions and/or underestimate the attractive ones being subject to relatively large uncertainties related to approximation in calculation of $U_{s t}$ and uncertainties in some physical constants $\left(A_{H}, k_{1}, k_{2}\right.$, cf. Eqs. (S-6), (S-7)). For instance, it is possible that iron oxide nanorods are not perfect cylinders but have a noncircular cross 
section, in which case van der Waals attraction is expected to be stronger than that predicted for cylindrical particles. Neutron scattering experiments are needed to carefully check this point.

Theoretical $U(h)$ dependencies of PCP samples, shown in Fig. 7b, exhibit a quantitatively different behavior from those of OPT samples. At the lowest considered free polymer concentration, $c_{w}=1 \mathrm{mg} / \mathrm{mL}$ (corresponding to $X \approx X_{o}$ ), the repulsive energy barrier progressively decreases with a decrease of the polymer grafting density from $+20 k_{B} T$ at the upper bound $n=0.4 \mathrm{~nm}^{-2}$ of the measured $n$-value and disappears at the lower bound $n=0.2$ $\mathrm{nm}^{-2}$ of the $n$-value, for which the overall interaction is attractive for all separations $h$. Similarly to OPT samples, at $n=0.4 \mathrm{~nm}^{-2}$ the secondary minimum becomes deeper and shifts to smaller separations $h$, while the repulsive energy barrier decreases with increasing free polymer concentrations $c_{w}$ up to $+1 k_{B} T$ at $c_{w}=14 \mathrm{mg} / \mathrm{mL}\left(X \approx 10 X_{o}\right)$ meaning that the thermal agitation energy is sufficient to overcome the potential barrier and make the particles fall into the deep primary minimum, meaning full destabilization of the suspension. Even though our theoretical estimation are subject to relatively large uncertainties, they clearly show that smaller grafting density of the PEG chains of PCP polymer leads to smaller equilibrium thickness of the adsorbed PCP layer ( $\delta=2.9-3.6 \mathrm{~nm}$ versus $\delta=6.1-7.7 \mathrm{~nm}$ for OPT, - see Table 1 - as evaluated by Eq. (S-8)) and, as a consequence, to much worse stability of the PCP samples as compared to OPT samples. Note that the value $\delta=6.1-7.7 \mathrm{~nm}$ for linear OPT molecules is rather close to that $(\delta=5-6 \mathrm{~nm})$ reported by Torrisi et al. ${ }^{23}$ for phosphonic acid PEG co-polymers grafted onto spherical iron oxide nanoparticles at nearly the same molecular weight of the PEG chains and at the PEG chain density $n \approx 1.5 \mathrm{~nm}^{-2}$.

\section{CONCLUSIONS}

This work has been focused on synthesis, characterization and colloidal stabilization of iron oxide nanorods of an average length $31 \mathrm{~nm}$ and an average diameter $6 \mathrm{~nm}$. The results of the present work can be summarized as follows:

1. Iron oxide nanorods were synthetized via two step route through combination of protocols proposed by Milosevic et al. ${ }^{17}$ and Mohapatra et al. ${ }^{4}$. Firstly, akaganeite nanorods were fabricated by hydrolysis of iron (III) salt at $80^{\circ} \mathrm{C}$ in the presence of PEI, used as capping agent. Secondly, akaganeite was reduced to iron oxide by hydrazine in a microwave reactor at $\sim 100^{\circ} \mathrm{C}$. Optimization of microwave parameters ( 3 cycles of $45 \mathrm{~s}$ at $200 \mathrm{~W}$ power) allowed obtaining rod-like particle morphology without appearance of polyhedral morphology. Our synthesis also presents an advantage of using relatively cheap reagents (PEI versus dopamine $\left.{ }^{17}\right)$ and moderate heating at the second step $\left(100^{\circ} \mathrm{C} \text { versus } 200^{\circ} \mathrm{C}\right)^{4}$.

2. Combination of PXRD, VSM and Mössbauer spectroscopy allows claiming that synthetized iron oxide nanorods contain about $11 \%$ of ordered ferrimagnetic magnetite or maghemite phase and about $89 \%$ of a disordered phase attributed to superparamagnetic magnetite or maghemite mixed with a disordered non-fully transformed akaganeite phase. Such a composition results in non-reversible magnetization curve, with remnant magnetization $M_{R} \approx 3.1 \mathrm{emu} / \mathrm{g}$ and a saturation magnetization $M_{S} \approx 13.4 \mathrm{emu} / \mathrm{g}$, which is $5-7$ times smaller than that of spherical maghemite nanoparticles.

3. Synthesized nanorods were dispersed in water after adsorption on their surface of three different polymers or polyelectrolytes allowing presumably steric stabilization, while electrostatic repulsion was screened by relatively high ion concentrations. Comparing OPT and PCP coatings with expected brush morphology, grafting density of PEG chains was shown to be crucial parameter affecting colloidal stability of nanorods. Higher values for OPT polymer, $n=2-4 \mathrm{~nm}^{-2}$ at saturation lead to much stronger steric repulsion and, consequently, much better colloidal stability of OPT samples as compared to PCP samples with $n=0.2-0.4$ 
$\mathrm{nm}^{-2}$ at nearly the same contour length of PEG chains of both polymers. For all the samples, the best colloidal stability (minimal hydrodynamic size) was obtained at an optimal concentration of added polymer $\left(X_{o}=0.5,1.3\right.$ and $1.6 \mathrm{mg} / \mathrm{mg}$ for PAA, PCP and OPT, respectively), coming from competition between increasing steric repulsion and increasing depletion attraction with an increase of adsorbed and free polymer concentrations, respectively.

4. The stability of PCP and PAA samples was strongly decreased with a decrease of solution $\mathrm{pH}$ below $\mathrm{pH} \approx 6$, that was tentatively explained by a change of the conformation of adsorbed polymer to a more compact one as its ionization degree decreases. On the contrary, solution $\mathrm{pH}$ (within the range $1-10$ ) did not significantly influence the stability of weakly charged OPT samples. A slightly better stability was obtained for OPT polymer grafted at $\mathrm{pH} \approx 1.2$ (OPT-1) rather than at $\mathrm{pH} \approx 6$ (OPT-6) likely because of stronger interactions between phosphonate groups and iron oxide surface at lower $\mathrm{pH}$. However even at the best conditions ( $X=X_{o}$, OPT-1 sample), the nanorods were still subject to some aggregation manifested through an increased effective hydrodynamic diameter $\left(d_{Z} \approx 60 \mathrm{~nm}\right.$ versus TEM size $L \times d=31 \times 6 \mathrm{~nm}$ ) and appearance of bundles composed of a few laterally stacked rods. Nevertheless, a significant progress towards iron oxide nanorod stability and its understanding seems to be achieved in the present work, as compared to the previously reported studies ${ }^{4,5,7,17,18}$.

5. Experimental conclusions on the effects of different parameters on nanorod colloidal stability were mostly supported by theoretical evaluation of the total energy of interaction between two nanorods. It was confirmed that nanorods coated with OPT polymer exhibit a stronger steric repulsion thanks to higher grafting density, while increase of the free polymer concentration in the suspending fluid leads to a deep secondary energy minimum $\left(\sim-10 k_{B} T\right)$ provoking nanorod aggregation. Intuitively, better repulsion between nanorods would be provided by adsorption of longer polymer chains but longer chains could result in a weaker grafting density, as reported for $\mathrm{PCP}^{45}$, such that a compromise between the chain length and grafting density has to be found.

Finally, the results of the present work could be useful for development of biomedical applications based on the use of rod-like magnetic nanoparticles, such as enhanced magnetic hyperthermia, MRI contrasting property and biological cell uptake, or as higher-sensitivity immunoassays, keeping in mind that these applications require a perfect colloidal stability of nanorods at the risk of the total loss of the nanorod performance in the case of colloidal aggregation.

\section{ASSOCIATED CONTENT:}

\section{Supporting information}

The Supporting Information is available free of charge on the ACS Publications website at DOI: XXX.

Characterization techniques (Sec. A); Supplementary results of nanorods characterization (Sec. B), including details of TEM analysis results (Sec. B-1), results of PXRD and Mössbauer spectroscopy (Sec. B-2), Details of characterization of polymer coated nanorods (Sec. B-3); Details of evaluation of energy of interaction between nanorods (Sec. C).

\section{AUTHOR INFORMATION}

\section{Corresponding author}

*E-mail: kuzhir@unice.fr. 


\section{ORCID}

Jéssica A. Marins: 0000-0002-3972-3957

Olivier Sandre: 0000-0002-1815-2702

Pavel Kuzhir: 0000-0001-7089-6197

\section{Funding}

P.K. and O.S. received funding for equipment from French "Agence Nationale de la Recherche" (project Future Investments UCAJEDI ref. $n^{\circ}$ ANR-15-IDEX-01 and project MagnetoChemoBlast, ref $\mathrm{n}^{\circ}$ ANR-13-BS08-0017); J.A.M received funding for postdoctoral fellowship from Brasilian "Ministério da Ciência, Tecnologia e Inovação - Conselho Nacional de Desenvolvimento Científico e Tecnológico" (CNPq, ref. $\mathrm{n}^{\circ}$ 203100/2014-0); A.P. received funding from German "Deutsche Forschungsgemeinschaft" (DFG, ref. n SE 2714/2-1).

\section{Notes}

The authors declare no competing financial interest.

\section{ACKNOWLEDGEMENTS}

The authors are grateful to J. Persello, A. Bée and D. Talbot for helpful discussions, S. Pagnota (UCA) for TEM imaging, G. Monge (Mines ParisTech) for XRD analyses, N. Guigo (UCA, ICN) for TGA, E. Briot for BET analysis, J.-M. Guigner for cryo-TEM (IMPMC, Sorbonne University) and P. Boustingorry for providing the polymers (Chryso).

\section{REFERENCES}

(1) Gupta, A. K.; Gupta, M. Synthesis and Surface Engineering of Iron Oxide Nanoparticles For Biomedical Applications. Biomaterials, 2005, 26, 3995-4021.

(2) Boyer, C., Whittaker, M. R., Bulmus, V., Liu, J., Davis, T. P. The Design and Utility of Polymer-Stabilized Iron-Oxide Nanoparticles for Nanomedicine Applications. NPG Asia Materials, 2010, 2, 23-30.

(3) Gossuin, Y., Disch, S., Vuong, Q. L., Gillis, P., Hermann, R. P., Park, J. H., Sailor, M. J. NMR Relaxation and Magnetic Properties of Superparamagnetic Nanoworms. Contrast Media \& Molecular Imaging, 2010, 5, 318-322.

(4) Mohapatra, J., Mitra, A., Tyagi, H., Bahadur, D., Aslam, M. Iron Oxide Nanorods as High-Performance Magnetic Resonance Imaging Contrast Agents. Nanoscale, 2015, 7, 9174-9184.

(5) Orza, A., Wu, H., Xu, Y., Lu, Q., Mao, H. One-Step Facile Synthesis of Highly Magnetic and Surface Functionalized Iron Oxide Nanorods for Biomarker-Targeted Applications. ACS Appl. Mate. Interfaces, 2017, 9, 20719-20727.

(6) Son, S. J., Reichel, J., He, B., Schuchman, M., Lee, S. B. Magnetic Nanotubes for Magnetic-Field-Assisted Bioseparation, Biointeraction, and Drug Delivery. J. Am. Chem. Soc., 2005, 127, 7316-7317.

(7) Das, R., Alonso, J., Nemati Porshokouh, Z., Kalappattil, V., Torres, D., Phan, M. H., Garaio E., García J.A., Sanchez Llamazares J.L., Srikanth, H. Tunable High Aspect Ratio Iron Oxide Nanorods for Enhanced Hyperthermia. The Journal of Physical Chemistry C, 2016, 120, 10086-10093.

(8) Wang, B. ; Bienvenu, C. ; Mendez-Garza, J. ; Lançon, P. ; Madeira, A. ; Vierling, P. ; Di Giorgio, C; Bossis, G. Necrosis of Hepg2 Cancer Cells Induced by the Vibration of Magnetic Particles. J. Magn. Magn. Mater., 2013, 344, 193-201.

(9) Leulmi, S.; Chauchet, X.; Morcrette, M.; Ortiz, G.; Joisten, H.; Sabon, P.; Livache, T.; Hou Y.; Carrière, M; Lequien S.; Dieny, B. Triggering the Apoptosis of Targeted Human Renal Cancer Cells by the Vibration of Anisotropic Magnetic Particles Attached to The Cell Membrane. Nanoscale 2015, 7, $15904-$ 15914.

(10) Chiriac H.; Radu E.; Țibu M.; Stoian G.; Ababei G.; Lăbușcă L.; Herea D.-D.; Lupu N. Fe-Cr-Nb-B Ferromagnetic Particles with Shape Anisotropy for Cancer Cell Destruction by Magnetomechanical Actuation. Sci. Rep. 2018, 8, 11538.

(11) Newman, D. M.; Heptinstall, J.; Matelon, R. J.; Savage, L.; Wears, M. L.; Beddow, J.; Cox, M.; Schallig, H.D.F.H.; Mens, P. F. A Magneto-Optic Route Toward the In Vivo Diagnosis of Malaria: Preliminary Results and Preclinical Trial Data. Biophys. J. 2008, 95, 994-1000. 
(12) Schrittwieser, S.; Pelaz, B.; Parak, W. J.; Lentijo-Mozo, S.; Soulantica, K.; Dieckhoff, J.; Ludwig, F.; Altantzis, T.; Bals, S.; Schotter, J. Homogeneous Protein Analysis by Magnetic Core-Shell Nanorod Probes. ACS Appl. Mater. Interfaces 2016, 8, 8893-8899.

(13) Schrittwieser, S.; Pelaz, B.; Parak, W. J.; Lentijo-Mozo, S.; Soulantica, K.; Dieckhoff, J.; Ludwig, F; Schotter, J. Direct Protein Quantification in Complex Sample Solutions By Surface-Engineered Nanorod Probes. Sci. Rep. 2017, 7, 4752.

(14) Newman, D; Matelon, R. Method and Device for Detecting an Analyte. U.S. Patent Application n. 14/241,534, 7 may 2015

(15) Ramzannezhad, A.; Gill, P.; Bahari, A. Fabrication of Magnetic Nanorods and Their Applications in Medicine. BioNanoMaterials, 2017, 18, 20170008.

(16) Cornel, R. M., and U. Shwertmann. Iron Oxides in the Laboratory. Preparation and Characterization; VCH Editions: Weinhein, 1991

(17) Milosevic, I.; Jouni, H.; David, C.; Warmont, F.; Bonnin, D.; Motte, L. Facile Microwave Process in Water for the Fabrication of Magnetic Nanorods. J. Phys. Chem. C 2011, 115, 18999-19004.

(18) Lentijo Mozo, S.; Zuddas, E.; Casu, A.; Falqui, A. Synthesizing Iron Oxide Nanostructures: The Polyethylenenemine (PEI) Role. Crystals, 2017, 7, 22.

(19) Choi, J.; Cha, J.; Lee, J-K. Synthesis of Various Magnetite Nanoparticles Through Simple Phase Transformation and their Shape-Dependent Magnetic Properties. RSC Adv., 2013, 3, 8365-8371.

(20) Blesa, M. A.; Mijalchik, M.; Villegas, M.; Rigotti, G. Transformation of Akaganeite into Magnetite in Aqueous Hydrazine Suspensions. Reactivity of solids, 1986, 2, 85-94.

(21) Luongo, G., Campagnolo, P., Perez, J. E., Kosel, J., Georgiou, T. K., Regoutz, A., Payne, D. J.; Stevens, M. M.; Ryan, M. P., Porter, A. E.; Dunlop, I. E. Scalable High-Affinity Stabilization of Magnetic Iron Oxide Nanostructures by a Biocompatible Antifouling Homopolymer. ACS Appl. Mater. Interfaces, 2017, 9, 40059-40069.

(22) Safi, M.; Sarrouj H.; Sandre, O.; Mignet, N.; Berret, J-F., Interactions between sub-10-nm Iron and Cerium Oxide Nanoparticles and 3T3 Fibroblasts: The Role of the Coating and Aggregation State. Nanotechnology 2010, 21, 145103 .

(23) Torrisi, V.; Graillot, A.; Vitorazi, L.; Crouzet, Q.; Marletta, G.; Loubat, C.; Berret, J. F. Preventing Corona Effects: Multiphosphonic Acid Poly (Ethylene Glycol) Copolymers for Stable Stealth Iron Oxide Nanoparticles. Biomacromolecules, 2014, 15, 3171-3179.

(24) Blin, T.; Kakinen, A.; Pilkington, E. H.; Ivask, A.; Ding, F.; Quinn, J. F.; Whittaker, M. R.; Chun Ke, P.; Davis, T. P. Synthesis and In Vitro Properties of Iron Oxide Nanoparticles Grafted with Brushed Phosphorylcholine and Polyethylene Glycol. Polym. Chem., 2016, 7, 1931-1944.

(25) Lalatonne, Y.; Paris, C., Serfaty, J-M.; Weinmann, P.; M. Lecouvey, L. Motte, Bis-Phosphonates-Ultra Small Superparamagnetic Iron Oxide Nanoparticles: A Platform towards Diagnosis and Therapy. Chem. Commun. 2008, 22, 2553-2555.

(26) Boyer, C., Bulmus, V., Priyanto, P., Teoh, W. Y., Amal, R., Davis, T. P. The Stabilization and BioFunctionalization of Iron Oxide Nanoparticles Using Heterotelechelic Polymers. J. Mater. Chem. 2009, 19, 111-123.

(27) Garofalo, A.; Parat, A.; Bordeianu, C.; Ghobril, C.; Kueny-Stotz, M.; Walter, A.; Jouhannaud, J.; BeginColin, S.; Felder-Flesch, D. Efficient Synthesis of Small-Sized Phosphonated Dendrons: Potential Organic Coatings of Iron Oxide Nanoparticles. New J. Chem., 2014, 38, 5226-5239.

(28) Basuki, J. S., Jacquemin, A., Esser, L., Li, Y., Boyer, C., Davis, T. P. A Block Copolymer-Stabilized CoPrecipitation Approach to Magnetic Iron Oxide Nanoparticles for Potential Use as MRI Contrast Agents. Polymer Chem. 2014, 5, 2611-2620.

(29) Basuki, J. S., Esser, L., Duong, H. T., Zhang, Q., Wilson, P., Whittaker, M. R., Haddleton, D.M, Boyer, C., Davis, T. P. (2014). Magnetic Nanoparticles with Diblock Glycopolymer Shells Give Lectin ConcentrationDependent MRI Signals and Selective Cell Uptake. Chem. Sci. 2014, 5, 715-726.

(30) Lam, T.; Avti, P. K.; Pouliot, P.; Tardif, J-C.; Rhéaume, É.; Lesage, F.; Kakkar A. Surface Engineering of SPIONs: Role of Phosphonate Ligand Multivalency in Tailoring their Efficacy. Nanotechnology 2016, 27, 415602.

(31) Bordeianu, C.; Parat, A.; Affolter-Zbaraszczuk, C.; Muller, R. N.; Boutry, S.; Begin-Colin, S.; Meyer, F.; Laurent, S.; Felder-Flesch, D. How a Grafting Anchor Tailors the Cellular Uptake and In Vivo Fate of Dendronized Iron Oxide Nanoparticles. J. Mater. Chem. B 2017, 5, 5152- 5164.

(32) Ahn, T.; Kim, J. H.; Yang, H-M.; Lee, J. W.; Kim, J-D. Formation Pathways of Magnetite Nanoparticles by Coprecipitation Method. J. Phys. Chem. C 2012, 116, 6069-6076.

(33) Sehgal, A.; Lalatonne, Y.; Berret, J. F.; Morvan, M. Precipitation- Redispersion of Cerium Oxide Nanoparticles with Poly (acrylic acid): Toward Stable Dispersions. Langmuir 2005, 21, 9359-9364. 